\title{
Antenna Systems and Electronic Warfare Applications. Edited by Richard A. Poisel, Artech House, 2012; 1036 pages. Price: £129.00, ISBN 978-1-60807-484-6
}

\author{
Shu-Kun Lin \\ MDPI AG, Kandererstrasse 25, CH-4057 Basel, Switzerland; E-Mail: lin@mdpi.com \\ Received: 10 January 2013 / Accepted: 14 January 2013 / Published: 17 January 2013
}

The following paragraphs are reproduced from the website of the publisher [1].

This comprehensive book serves as a one-stop resource for practical EW antenna system know-how. Supported with over 700 illustrations and nearly 1,700 equations, this authoritative reference offers you detailed explanations of all the important foundations and aspects of this technology. Moreover, you get an in-depth treatment of a wide range of antenna system applications.

The book presents the key characteristics of each type of antenna, including dipoles, monopoles, loops, arrays, horns, and patches. This authoritative volume enables you to analyze and design broadband communication and radar EW antennas, interface antennas to receivers and power amplifiers with maximum efficiency, use multicouplers to connect multiple receivers to a single antenna, and apply the correct kind of antenna to EW problems.

\section{Table of Contents}

Preface

Chapter 1. Introduction to electronic warfare antenna systems

Chapter 2. Principles of electromagnetic radiation

Chapter 3. Fundamental antenna properties

Chapter 4. Transmission lines

Chapter 5. Dipole antennas

Chapter 6. Monopole antennas

Chapter 7. Loop antennas

Chapter 8. Traveling wave antennas

Chapter 9. Antenna arrays

Chapter 10. EW applications of antenna arrays 
Chapter 11. Yagi-Uda antennas

Chapter 12. Frequency independent $\mathrm{EW}$ antennas

Chapter 13. Aperture antennas

Chapter 14. Electrically small EW antennas

Chapter 15. Patch antennas

Chapter 16. EW applications of patch antennas

Chapter 17. Adaptive EW antenna arrays

Chapter 18. Fractal antennas

Chapter 19. Genetically designed EW antennas

Chapter 20. Antenna matching

Chapter 21. Multicouplers, combiners, and diplexers

Chapter 22. Radomes

Appendix A. RF amplifiers

Appendix B. RF switches

Appendix C. The method of moments

Appendix D. Properties of dielectric materials.

\section{List of Acronyms}

About the Author

Index

* Editor's Note: The brief summary and the contents of the books are reported as provided by the authors or the publishers. Authors and publishers are encouraged to send review copies of their recent books of potential interest to readers of Sensors to the Publisher (Dr. Shu-Kun Lin, Multidisciplinary Digital Publishing Institute (MDPI), Kandererstrasse 25, CH-4057 Basel, Switzerland. Tel. +41-61-683-77-34; Fax: +41-61-302-89-18; E-Mail: lin@mdpi.com). Some books will be offered to the scholarly community for the purpose of preparing full-length reviews.

\section{Note}

1. The website for this book is: http://www.artechhouse.com/International/Books/Antenna-Systemsand-Electronic-Warfare-Application-1947.aspx.

(C) 2013 by the authors; licensee MDPI, Basel, Switzerland. This article is an open access article distributed under the terms and conditions of the Creative Commons Attribution license (http://creativecommons.org/licenses/by/3.0/). 